\title{
Clapping in Delaminated Sandwich-beams due to Forced Oscillations
}

\author{
I. Müller \\ Universität Karlsruhe, Institut für Mechanik
}

Institut für Mechanik

Kaiserstr. 12, Geb. 20.30

76128 Karlsruhe

Tel.: +49 (0) 721/ 608-2071

Fax: +49 (0) 721/ 608-7990

E-Mail: ifm@uni-karlsruhe.de

www.ifm.uni-karlsruhe.de 


\title{
Clapping in Delaminated Sandwich-beams due to Forced Oscillations
}

\author{
I. Müller \\ Institut für Mechanik \\ Universität Karlsruhe $(\mathrm{TH})$ \\ D-76131 Karlsruhe, Germany \\ e-mail: imueller@ifm.uni-karlsruhe.de
}

\begin{abstract}
Forced oscillations of delaminated composite laminates lead to non-smooth dynamic systems due to continuously developing impact-like contacts along the delamination. The primary aim of this study is to investigate a realistic model situation for delamination problems based on experiments and numerical simulation. First, the properties of lateral impacts on beam-type structures are studied, in particular in regard to the amount of energy dissipation during contact. Taking these results into account the Finite Element method based on beam elements is employed to simulate the stationary state of typical oscillations observed on the delaminated structure paying special attention to the dynamically developing and strongly dissipative contact. To deal with that kind of contact arising between delaminated layers, an effective technique combining a law of impact with the classical penalty method is presented. Confronting numerical results with the experimental reference provides an insight into the accuracy and robustness of the simulation technique.
\end{abstract}

Keywords. nonlinear oscillations, lateral impacts, damage detection.

\section{Introduction}

Sandwich structures are widely employed in the engineering practice. Correspondingly, a major interest lies in non-destructive testing of the structural integrity, particularly in relation to the occurrence of delaminations between adjacent plies as one of the most common failure modes in composite laminates. Delamination may be caused by impact loads, fatigue or poor fabrication. Basically, there are two different testing procedures, acoustic or ultrasonic methods and vibration-based methods. The first ones are localized experimental methods which require that the vicinity of the damage is known a priori and that the portion of the structures being inspected is readily accessible [1]. Some of them are impractical for specimens with large geometrical dimensions [2]. The limitation relates to the sensitivity of the detection method. Since the driving is relatively small, the required high power continuous wave is usually difficult to achieve [3]. Therefore, the need for quantitative global damage detection methods leads to vibration based methods. One of the main advantages of such methods is the nearly unlimited driving power which allows damage to be identified not only locally but globally at any place on the structure. Currently available vibration-based methods are mostly linear methods, since these methods take into account property changes in linear dynamic systems (resonance frequencies, modal characteristics etc.) to detect the simple presence of a delamination. A reliable prognosis of the size and the position of delamination is not successful in the majority of cases.

Investigations of the nonlinear response are promising for localization and to quantify the size of the damage on a global basis. The nonlinearity arises from a local contact phenomena, clapping. The delaminated layer and the remaining part of a sandwich structure periodically strike against one another during the oscillation. This dynamic contact-impact problem has as the simplest approximation two pendulums which are simultaneously excited by harmonic base excitation [4]. Depending on the frequency and amplitude of excitation, a cascade of bifurcations with intermittent windows of irregular motions and different numbers of impacts during one excitation period can occur. The essential point in this scenario is its dependency on the amount of energy dissipation at each impact. 
In the following, a more realistic situation will be considered. The delaminated sandwich beam consists of two separated laminae with different cross sections. In the considered case, the upper ends are free, the lower ends are in permanent contact and clamped. In the static equilibrium state there is a gap between both lamina. The forcing is a harmonic base excitation. First, experiments are performed to provide a realistic reference for the oscillation behavior dominated by the continuously evolving lateral contact. Second, this dynamic impact problem is investigated. Finally, a mechanical model for this continuous system is proposed based on discretization into a system with multi degrees of freedom derived by Finite Element methods.

\section{Experimental Investigations}

\subsection{Mechanical system and experimental arrangement}

The mechanical system under consideration is depicted in figure 1 [a]. It consists of two laminae, whose shapes distinctly deflect from an ideal straight line in the statical stress-free state.
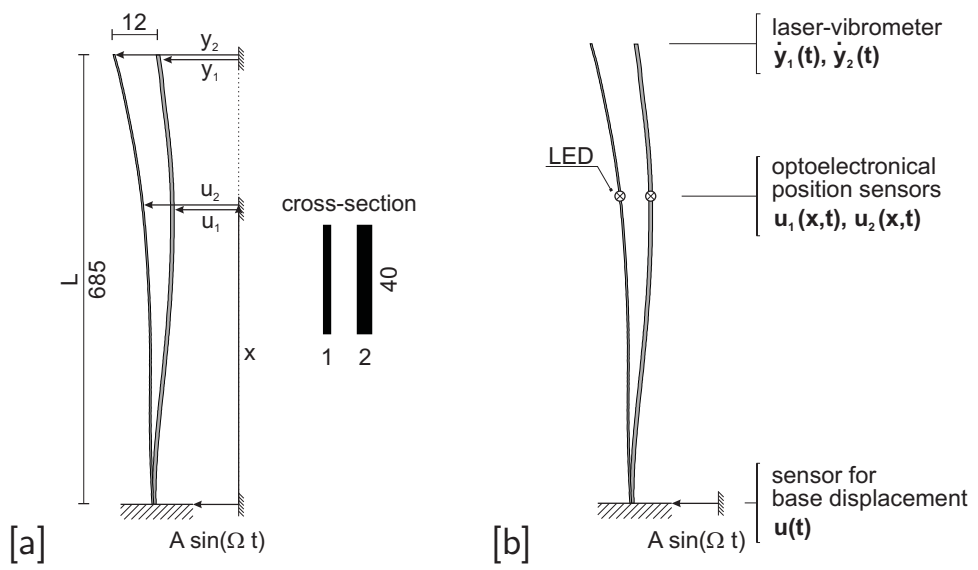

Fig. 1: Mechanical system: [a] geometry, [b] sensors.

Both laminae of length $685 \mathrm{~mm}$ are clamped at the lower ends. The upper ends are free. The maximum width of the gap at the upper ends at rest is $12 \mathrm{~mm}$. Both laminae are made from aluminium with slightly different material properties (density: $\varrho_{1}=2680 \mathrm{~kg} / \mathrm{m}^{3}, \varrho_{2}=2760 \mathrm{~kg} / \mathrm{m}^{3}$ and Y OUNG's modulus $\left.E_{1}=5.85 \cdot 10^{10} \mathrm{~N} / \mathrm{m}^{2}, E_{2}=4.61 \cdot 10^{10} \mathrm{~N} / \mathrm{m}^{2}\right)$. The cross-sections are $2 \times 40 \mathrm{~mm}$ (thick lamina) and $1 \times 40 \mathrm{~mm}$ (thin lamina), respectively. The displacements $q_{1}(x, t)$ and $q_{2}(x, t)$ at the distance $x$ along the vertical at time $t$ are absolute quantities. The base excitation $A \sin (\Omega t)$ is harmonic with amplitude $A$ and driving angular frequency $\Omega$. In the following, the amplitude $A=2.5 \mathrm{~mm}$ will be kept constant and only the frequency $\Omega=2 \pi f$ will be changed.

To characterize the properties of oscillations several sensors are used. Two optoelectronic position sensors give the distance of two corresponding LEDs to a vertical reference. In this way, the initial shape $u_{1,0}(x)$ and $u_{2,0}(x)$ of both laminae and the displacements $y_{1}(t)=u_{1}(L, t)$ and $y_{2}(t)=$ $u_{2}(L, t)$ at the top will be monitored. For some selected excitation frequencies the deflection curves $u_{1}(x, t)$ and $u_{2}(x, t)$ during the oscillation are captured. Additionally, a laser vibrometer measures the velocity $\dot{y}_{1}(t)$ and $\dot{y}_{2}(t)$ at the upper end of both laminae. A position sensor at the lower end controls the amplitude of excitation $A$. 


\subsection{System response on harmonic excitation}

To get a first impression about the nonlinear properties, the frequency response curves of both laminae are measured. For this, a range of $0.5 \mathrm{~Hz} \leq f \leq 4.0 \mathrm{~Hz}$ was selected for the frequency of base excitation, since the lowest natural frequencies of both laminae can be found in this band. For harmonic excitation, a sweep sine function with duration of $30 \mathrm{~min}$ was applied. This kind of excitation ensures a sufficiently stationary response.

Figure 2 [a] exhibits the linear behavior of both laminae. Each lamina is investigated separately for this purpose. The state variables $\bar{y}_{1}(t), \bar{y}_{2}(t)$ denote the dynamic displacement at the top of each lamina counted from the static curved state, respectively:

$$
\bar{y}_{1}(t)=y_{1}(t)-u_{1,0}(L), \quad \bar{y}_{2}(t)=y_{2}(t)-u_{2,0}(L) .
$$

As expected, each subsystem shows a typical resonance curve with large amplitudes at resonance. The corresponding lowest natural frequencies are $1.47 \mathrm{~Hz}$ and $3.17 \mathrm{~Hz}$, respectively.

[a]
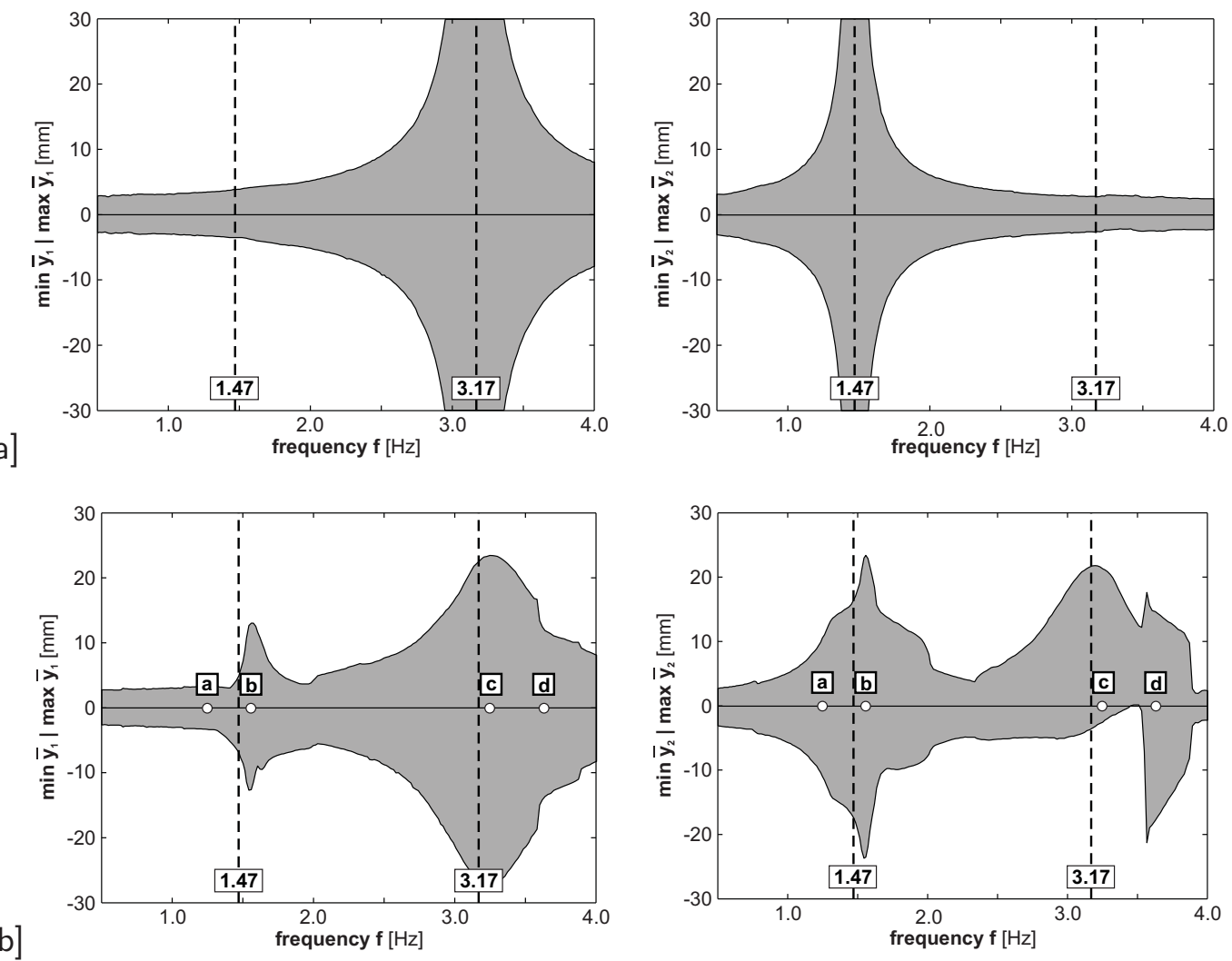

Fig. 2: Experimentally gained frequency response curves of both laminae (left column: thick lamina, right column: thin lamina): [a] without contact, [b] in contact.

Figure 2[b] illustrates the influence of nonlinear dynamic contact problem which is especially indicated by continuous evolution of the contact line. Due to the coupling of the subsystems, the parts interact with each other. Thus, the amplitudes at resonance of the linear subsystems are reduced significantly. At this point it is evident that contact damping has a major influence on the shape of the oscillation. This fact is illustrated by four stationary time-displacement responses with different frequencies of excitation (the used values $f=\{1.25 \mathrm{~Hz}, 1.60 \mathrm{~Hz}, 3.25 \mathrm{~Hz}, 3.65 \mathrm{~Hz}\}$ correspond to the white dots [a] - [d] in Figures 2 [b]). 
[a]
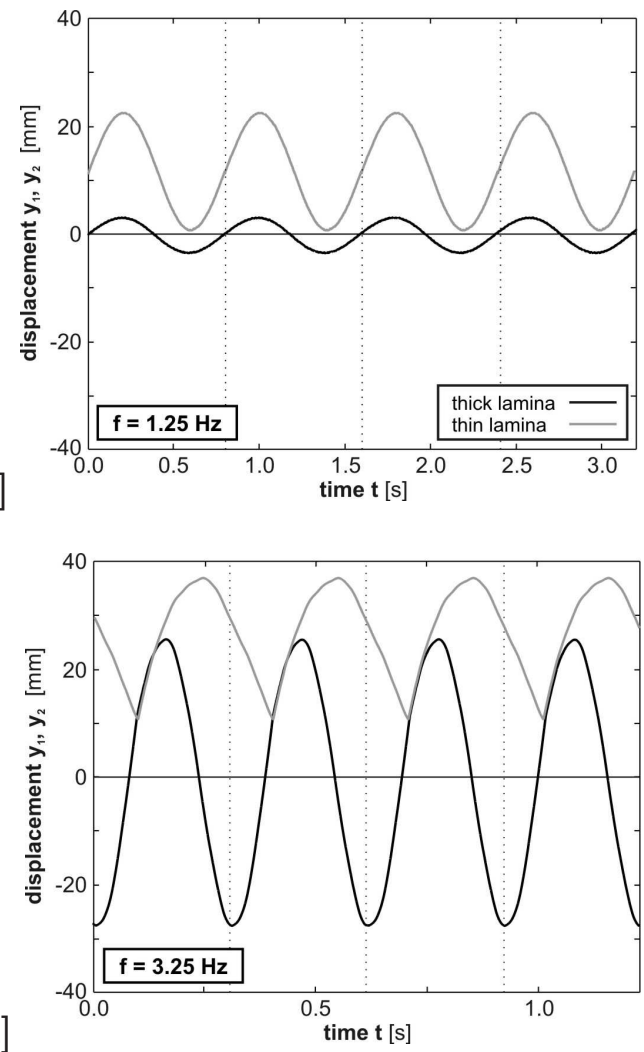

[b]
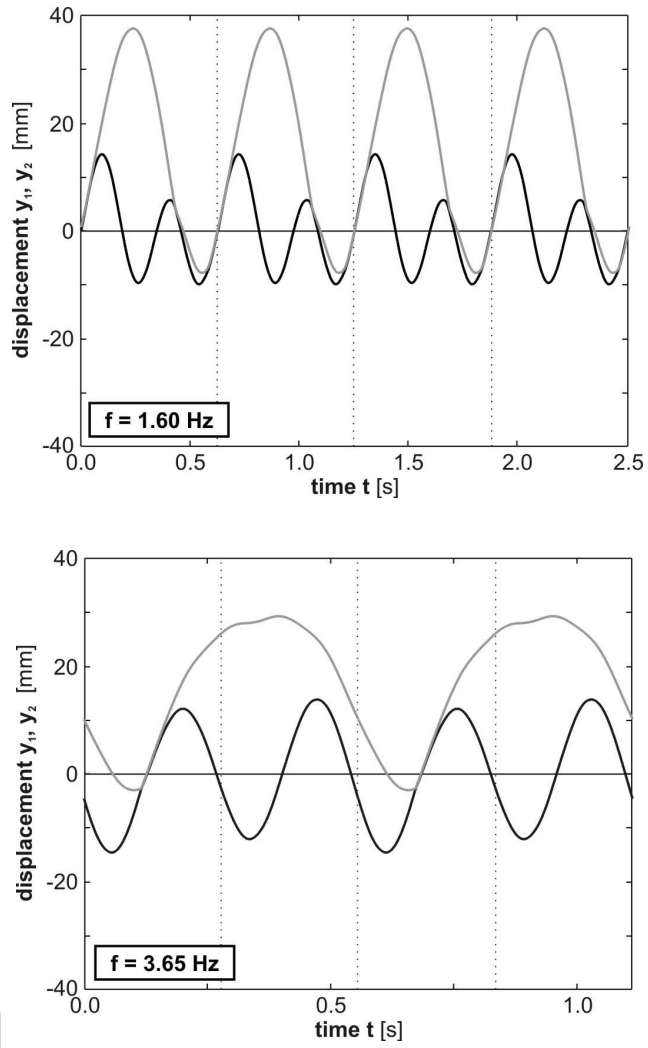

Fig. 3: Experimentally gained time-displacement curves for different excitation frequencies and constant amplitudes.

Excitation with $f=1.25 \mathrm{~Hz}$ gives almost harmonic responses with the same frequency as the excitation. No contact occurs during the course of time at the tip of the laminae. However, near the clamped ends contacts with weak intensity may arise although they hardly affect the oscillation properties at the tip. Despite this fact, slightly nonlinear responses can be determined at top position. The value $f=1.60 \mathrm{~Hz}$ lies in the vicinity of the resonance of the subsystem with coordinate $y_{2}$ (thin lamina). As can be seen in figure 3 [b], the thick lamina is beaten by the thin one. In contrast, $f=3.25 \mathrm{~Hz}$ (figure $3[\mathrm{c}]$ ) is located in the vicinity of the resonance of the subsystem with coordinate $y_{1}$ (thick lamina). Hence, the thick lamina beats the thin one. In both cases the beating can be heard acoustically as a clapping noise. Finally, an excitation frequency $f=3.65 \mathrm{~Hz}$ lies within a window of bifurcated oscillations. Period doubling occurs in such a way that one contact in two excitation periods can be found.

To explore the phenomenon of a continuously evolving contact line during the oscillation in more detail, Figure 4 depicts several states of the deflection shape during one period of excitation $T$. In fact, the contact duration is not constant for specific points along the beam axis. Despite this fact, the only considered point at the tip of the beams is supposed to be representative for the total motion. 


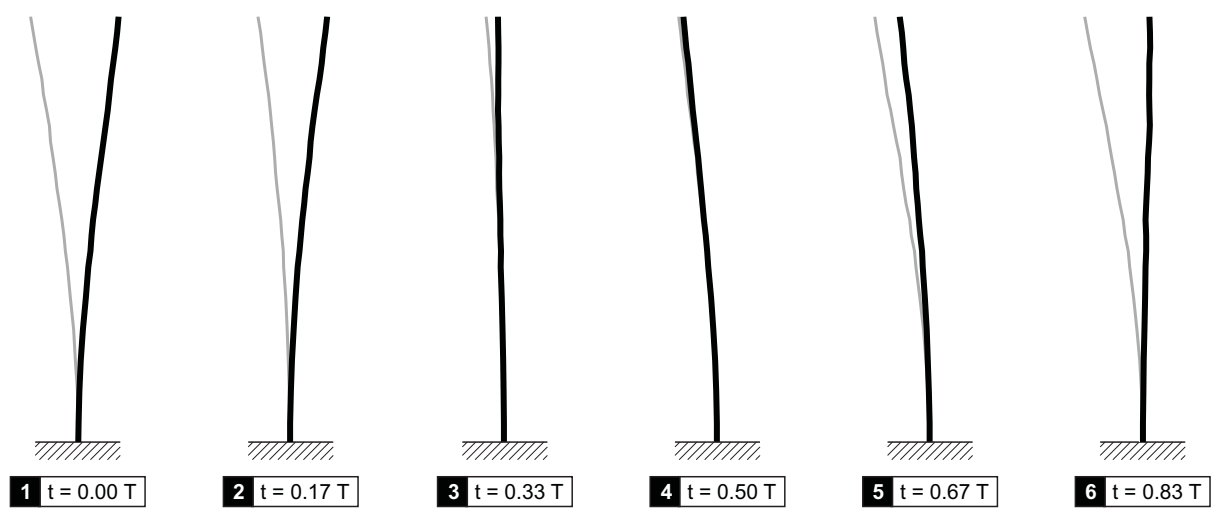

Fig. 4: Experimentally gained operation deflection shape during one excitation period $T, f=3.25 \mathrm{~Hz}$.

In view of the experimental identification of delamination, the oscillation with $f=3.25 \mathrm{~Hz}$ is the preferable one. Both contacting points exhibit opposite velocities directly before contact arises. The resulting impact is the maximum strike compared to all other frequencies of excitation. The response spectrum of amplitudes shows approximately a harmonic response for the thick lamina that is in resonance. In contrast, the response of the thin lamina contains a distribution of higher harmonics of excitation frequency (Fig. 5 [b]). This fact indicates the presence of a nonlinear response.

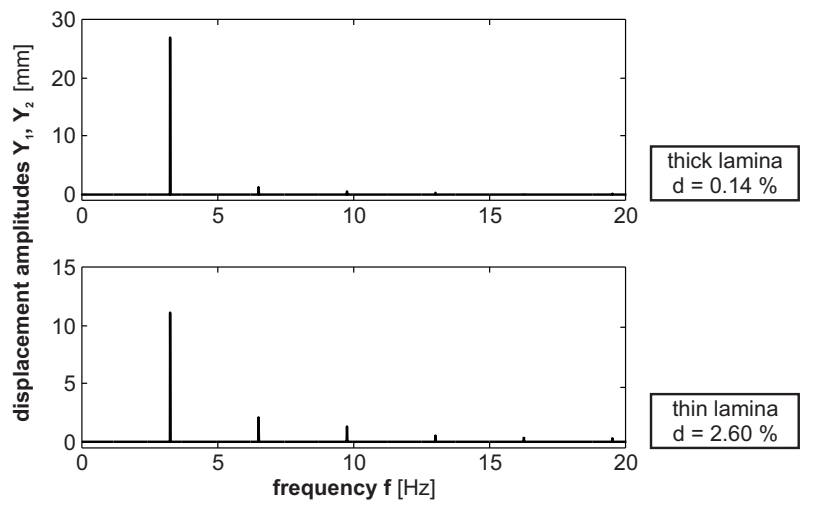

Fig. 5: Response spectra of displacement amplitudes for excitation frequency $f=3.25 \mathrm{~Hz}$ : thick lamina (upper), thin lamina (lower).

The distortion factor

$$
d=\sqrt{\frac{\sum_{i=1}^{n} A_{i}{ }^{2}}{A_{1}{ }^{2}}}-1
$$

compares the amplitudes $A_{i}$ of the higher harmonics with the amplitude $A_{1}$ of the basic oscillation. This procedure enables us to measure the nonlinearity of the system's response. The quantity of the distortion factor is well-known in the science of electrical engineering (e.g. [5]) and can be transferred to the nonlinear mechanical problem. The considered oscillation has $d_{1}=0.14 \%$ for the thick lamina and $d_{2}=2.60 \%$ for the thin one. It should be emphasized here, the corresponding spectra for the velocities or even for the accelerations would more highlight the result of a highly nonlinear response for the thin lamina.

As can be seen (Fig. 3, [b]-[d]), the time interval of contact during one period of excitation is relatively large. Such a phase of common motion of both laminae is only possible if the initial impact, which 
initiates the contact phase, is highly dissipative. Otherwise, both laminae would separate immediately. In the following, the considerations must be focussed on the investigation of the impact properties.

\subsection{Lateral impact on a beam}

One-dimensional continua are rods and beams. Wave propagation in rods induced by longitudinal impacts have been investigated intensively (e.g. [6]). In the present case, lateral impacts on beams are of interest. This type of contact, in particular a continuously developing line of contact involved, has not yet attracted considerable attention. The main intention is to find information about energy dissipation. For this purpose, two fundamental experiments corresponding to extreme cases were performed.

A straight elastic beam (lamina) made of steel is clamped at one end on a horizontal surface of high rigidity. In the stress-free state the beam touches the surface at every point along its center line. The experiment starts by lifting the free end up a certain distance from the surface. After release, the beam impacts the surfaces in a rolling motion accompanied by a continuous evolution of the contact line from the clamped end to the free one. Such continuous contact is very different from the well-known punctual contacts. One of the main properties is the total dissipation of the energy into the surface. No snap-back or local lift off occurs in the time scale of the impact.

In a second experiment, only the free end of the beam touches a stiff block of brass in the stress-free state. Here, a piezo-electric force transducer measures the contact force. Pairs of strain-gauges are applied in the vicinity of the support and at some distance (see Fig. 6) to achieve the strain wave along the beam axis.

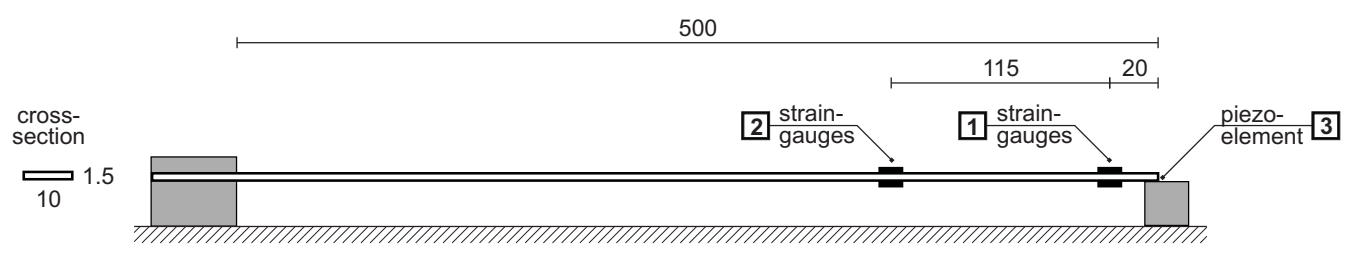

Fig. 6: Experimental setup.

The free tip of the beam is lifted up $50 \mathrm{~mm}$ and is then released. The impact force (sensor 3 ) is dominated by a large peak with a duration of about $30 \mu \mathrm{s}$. This time interval is extremely small compared to the period of about $50 \mathrm{~ms}$ at the lowest natural frequency of the beam. Again, no snap back of the system occurs in the time scope of the impact. In an enlarged time range of the ensuing oscillation a snap-back effect cannot be excluded. Applying the time scale of bending oscillations, the impact can be considered to arise suddenly. Considering the strain wave (sensors 1 and 2), its maximum amplitude decay and the signal becomes distorted with increasing distance from the point of impact due to energy radiation. It can be proved that the mean wave velocity is much smaller than in the case of a pure longitudinal wave problem. The distinct energy dissipation is associated with two major mechanisms: the progressional radiation on the surface of the beam due to transversal wave components and a measurable heat production in the contact interface. The latter one can be observed by a thermographic analysis. 

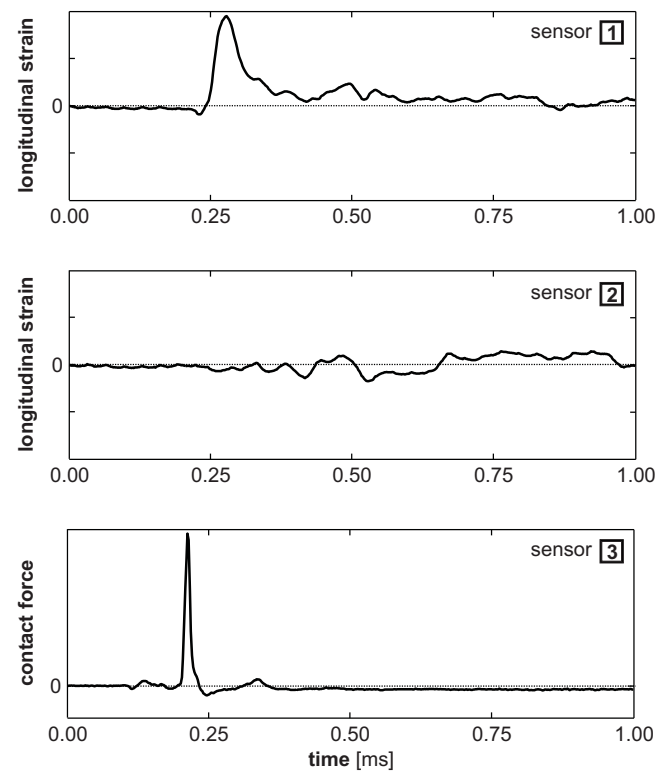

Fig. 7: Signals for longitudinal strain [1], [2] and contact force [3].

Summarizing the facts, the treated type of contact is characterized by vanishing contact time compared to the time scale of the bending oscillation and strong energy dissipation during the contact event.

Recalling the system with two contacting laminae as an example for oscillations of delaminated structures, the corresponding dynamic contact problem shows similar features as the one previously described. Only the time scale must be enlarged. Figure 8 captures the velocities $\dot{y}_{1}(t)$ and $\dot{y}_{2}(t)$ of both laminae within two periods of excitation with $f=3.25 \mathrm{~Hz}$.
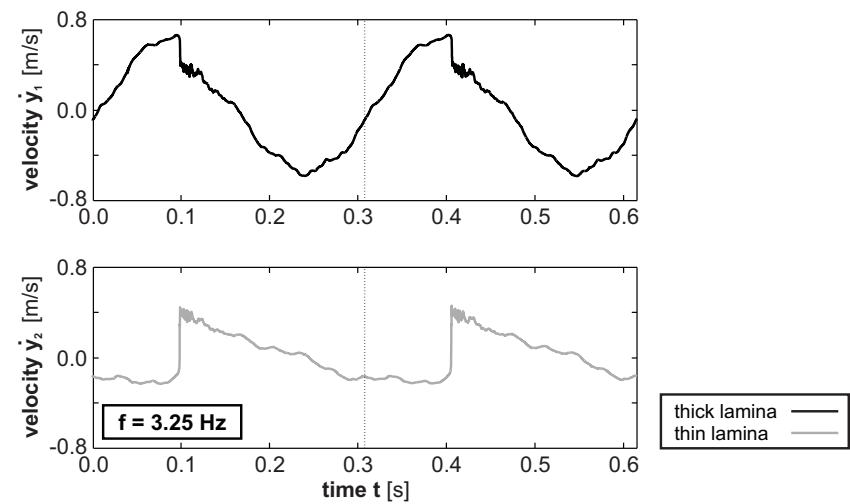

Fig. 8: Impact-dominated velocities of both laminae at the free ends in the delamination problem.

The discontinuity (jump) in the velocities at the beginning of contact on the considered point is clearly visible. It is evident that this phenomenon can be described by classical impact theory. This theory links the relative velocity $\dot{Q}\left(t^{\langle c\rangle}-0\right)=\dot{q}_{u}\left(t^{\langle c\rangle}-0\right)-\dot{q}_{v}\left(t^{\langle c\rangle}-0\right)$ of the impacting masses $m_{u}$, $m_{v}$ before contact and the relative velocity $\dot{Q}\left(t^{\langle c\rangle}+0\right)=\dot{q}_{u}\left(t^{\langle c\rangle}+0\right)-\dot{q}_{v}\left(t^{\langle c\rangle}+0\right)$ after contact by a coefficient of restitution $e$

$$
\dot{Q}\left(t^{\langle c\rangle}+0\right)=-e \dot{Q}\left(t^{\langle c\rangle}-0\right)
$$


The corresponding coefficient of restitution $0 \leq e \leq 1$ depends on the amount of energy dissipation at contact time $t^{\langle c\rangle}$. The coefficient of restitution accommodates the ratio of the impulse of expansion and the impulse of compression which occur during the contact phase. Thus, for all values of restitution $e<1$ an impact dissipation is involved. To get the coefficient of restitution, the velocities $\dot{y}_{1}\left(t^{\langle c\rangle}-0\right), \dot{y}_{2}\left(t^{\langle c\rangle}-0\right)$ before impacting at the tip and $\dot{y}_{1}\left(t^{\langle c\rangle}+0\right), \dot{y}_{2}\left(t^{\langle c\rangle}+0\right)$ after the impact are taken from the experiment. The ratio of masses is approximately given by the ratio of cross-sections as $m_{1} / m_{2}=1 / 2$. Within the scope of the experimentally gained data, the well-known formulas of NEWTON's impact law

$$
\begin{aligned}
& \dot{y}_{1}\left(t^{\langle c\rangle}+0\right)=\dot{y}_{1}\left(t^{\langle c\rangle}-0\right)-(1+e) \frac{m_{2}}{m_{1}+m_{2}} \dot{Y}\left(t^{\langle c\rangle}-0\right), \\
& \dot{y}_{2}\left(t^{\langle c\rangle}+0\right)=\dot{y}_{2}\left(t^{\langle c\rangle}-0\right)+(1+e) \frac{m_{1}}{m_{1}+m_{2}} \dot{Y}\left(t^{\langle c\rangle}-0\right),
\end{aligned}
$$

reveal a range of $0.0 \leq e \leq 0.1$ for the restitution coefficient. This result confirms the large contact damping arising at lateral impacts on beams, whose origin was previously discussed.

\section{Computation by Finite Element Methods}

For the numerical calculation, the one-dimensional continuum problem is transformed into two multiDOF systems by FE methods. Both laminae are discretized by the same number of EULER-BERNOULLI beam elements. This leads to the equations of motion as two linear systems with $2 n$ DOF

$$
\begin{aligned}
& \mathbf{M}_{1} \ddot{\mathbf{q}}_{\mathbf{1}}(t)+\mathbf{C}_{\mathbf{1}} \dot{\mathbf{q}}_{\mathbf{1}}(t)+\mathbf{K}_{\mathbf{1}} \mathbf{q}_{\mathbf{1}}(t)=\mathbf{f}_{\mathbf{1}}(t), \\
& \mathbf{M}_{\mathbf{2}} \ddot{\mathbf{q}}_{\mathbf{2}}(t)+\mathbf{C}_{\mathbf{2}} \dot{\mathbf{q}}_{\mathbf{2}}(t)+\mathbf{K}_{\mathbf{2}} \mathbf{q}_{\mathbf{2}}(t)=\mathbf{f}_{\mathbf{2}}(t)
\end{aligned}
$$

and the appropriate state variables

$$
\begin{aligned}
& \mathbf{q}_{\mathbf{1}}=\left[q_{1,1}, q_{1,2}, \ldots, q_{1, n}\right]^{T}, \\
& \mathbf{q}_{\mathbf{2}}=\left[q_{2,1}, q_{2,2}, \ldots, q_{2, n}\right]^{T} .
\end{aligned}
$$

The generalized coordinates given in eq. (6) can be subdivided into translatory and rotational DOFs leading to the displacements $u$ and the rotations $\varphi$

$$
\mathbf{q}_{\mathbf{i}}=\left[u_{i, 1}, \varphi_{i, 1}, \ldots, u_{i, n / 2}, \varphi_{i, n / 2}\right]^{T} \quad \text { with } y_{i}=u_{i, n / 2}, \quad i=1(1) 2 .
$$

A prescribed displacement as a harmonic base excitation is applied to the lowest node of each subsystem. Additionally, a clamped support is assumed on these nodes.

$$
\mathbf{q}_{\mathbf{i}, \mathbf{a}}=\left[\begin{array}{l}
q_{i, 1} \\
q_{i, 2}
\end{array}\right]=\left[\begin{array}{l}
u_{i, 1} \\
\varphi_{i, 1}
\end{array}\right]=\left[\begin{array}{c}
A \sin (\Omega t) \\
0
\end{array}\right], \quad i=1(1) 2
$$

A partitioning of the set of equations (eq. (5)) in consideration of the prescribed coordinates $\ddot{\mathbf{q}}_{\mathbf{i}, \mathbf{a}}$, $\dot{\mathbf{q}}_{\mathbf{i}, \mathbf{a}}, \mathbf{q}_{\mathbf{i}, \mathbf{a}}$ and the unknown nodal quantities $\ddot{\mathbf{q}}_{\mathbf{i}, \mathbf{b}}, \dot{\mathbf{q}}_{\mathbf{i}, \mathbf{b}}, \mathbf{q}_{\mathbf{i}, \mathbf{b}}$ yields

$$
\begin{aligned}
& {\left[\begin{array}{ll}
\mathbf{M}_{\mathbf{i}, \mathbf{a a}} & \mathbf{M}_{\mathbf{i}, \mathbf{a b}} \\
\mathbf{M}_{\mathbf{i}, \mathbf{b a}} & \mathbf{M}_{\mathbf{i}, \mathbf{b b}}
\end{array}\right]\left[\begin{array}{c}
\ddot{\mathbf{q}}_{\mathbf{i}, \mathbf{a}} \\
\ddot{\mathbf{q}}_{\mathbf{i}, \mathbf{b}}
\end{array}\right]+\left[\begin{array}{ll}
\mathbf{C}_{\mathbf{i}, \mathbf{a a}} & \mathbf{C}_{\mathbf{i}, \mathbf{a b}} \\
\mathbf{C}_{\mathbf{i}, \mathbf{b a}} & \mathbf{C}_{\mathbf{i}, \mathbf{b b}}
\end{array}\right]\left[\begin{array}{c}
\dot{\mathrm{q}}_{\mathbf{i}, \mathbf{a}} \\
\dot{\mathbf{q}}_{\mathbf{i}, \mathbf{b}}
\end{array}\right]+\left[\begin{array}{cc}
\mathbf{K}_{\mathbf{i}, \mathbf{a a}} & \mathbf{K}_{\mathbf{i}, \mathbf{a b}} \\
\mathbf{K}_{\mathbf{i}, \mathbf{b a}} & \mathbf{K}_{\mathbf{i}, \mathbf{b b}}
\end{array}\right]\left[\begin{array}{c}
\mathbf{q}_{\mathbf{i}, \mathbf{a}} \\
\mathbf{q}_{\mathbf{i}, \mathbf{b}}
\end{array}\right]=\left[\begin{array}{c}
\mathbf{f}_{\mathbf{i}, \mathbf{a}} \\
\mathbf{f}_{\mathbf{i}, \mathbf{b}}
\end{array}\right]} \\
& i=1(1) 2
\end{aligned}
$$


As a result of the second row the unknown response quantities $\ddot{\mathbf{q}}_{\mathbf{i}, \mathbf{b}}, \dot{\mathbf{q}}_{\mathbf{i}, \mathbf{b}}, \mathbf{q}_{\mathbf{i}, \mathbf{b}}$ are obtained

$$
\begin{aligned}
& \mathbf{M}_{\mathbf{i}, \mathbf{b b}} \ddot{\mathbf{q}}_{\mathbf{i}, \mathbf{b}}+\mathbf{C}_{\mathbf{i}, \mathbf{b b}} \dot{\mathbf{q}}_{\mathbf{i}, \mathbf{b}}+\mathbf{K}_{\mathbf{i}, \mathbf{b b}} \mathbf{q}_{\mathbf{i}, \mathbf{b}}=\mathbf{f}_{\mathbf{i}, \mathbf{b}}-\mathbf{M}_{\mathbf{i}, \mathbf{b a}} \ddot{\mathbf{q}}_{\mathbf{i}, \mathbf{a}}-\mathbf{C}_{\mathbf{i}, \mathbf{b a}} \dot{\mathbf{q}}_{\mathbf{i}, \mathbf{a}}-\mathbf{K}_{\mathbf{i}, \mathbf{b a}} \mathbf{q}_{\mathbf{i}, \mathbf{a}}, \\
& i=1(1) 2
\end{aligned}
$$

which are of particular interest.

Coupling of both subsystems, represented by the two sets of equations (5), occurs because of several lateral contact events along the longitudinal axis of the beams. This fact causes the strong nonlinearity of the problem. The continuous evolution of the contact line must be separated into several nodeto-node contacts of opposite nodes. During the oscillation the succession of contact points and the corresponding time when contacts occur are unknown a priori. These are typical features of a nonsmooth dynamic system.

Standard procedures in FE methods for contact modeling are the Augmented-LAGRANGE method and the penalty method. The main problem of both methods is the inclusion of the strongly dissipative character of the dynamic contact. Applications of the first procedure are not known in this field. The classical penalty method can be extended by introducing penalty damping in addition to the penalty stiffness. Thus, this procedure requires the choice of two penalty parameters, which have no physically specifiable quantity [7]. Moreover, these parameters are mutually dependent with the time step of integration [10]. The arising numerical sensitivity of this method was investigated in previous works [10], [11]. A parameter estimation is only possible by comparing numerical results with an experimental reference for stationary oscillations [11]. In the following, two different approaches both based on NEWTON's impact law are presented aimed at discovering a robust and efficient method for description of strong dissipative dynamic contact within the simulation technique.

\subsection{NeWTON's impact law for contact description within FE methods}

Sudden impacts accompanied by strong energy dissipation can be modeled by the classical theory of impact in which the velocities during a contact event are controlled by a law of impact, e.g. N EWTON's impact law. This procedure, which has been proven in the field of rigid body dynamics, can be adopted for description of dissipative contact within the Finite Element methods. Applying an impact law, sudden change of the velocities of the contact nodes within a vanishing time interval is assumed. Moreover, during the impact the position of the contact nodes remains constant. At each pair of contact nodes the law of impact must be applied and only one parameter is needed, namely the coefficient of restitution, which is known from the preceding experimental investigation. Thus, the numerical procedure is an extension of the ideas for a 2 DOF system [4].

Supplying the harmonic base excitation on the treated system, coupling of both linear subsystems occurs due to impact-like contact events along the beam. Each contact gives rise to a discontinuity of the system's behavior. The integration of such non-smooth dynamic system leads to a sequence of smooth systems, whose solutions must be patched together on those points in time $t^{\langle j\rangle}$ when an irregularity due to contact occurs

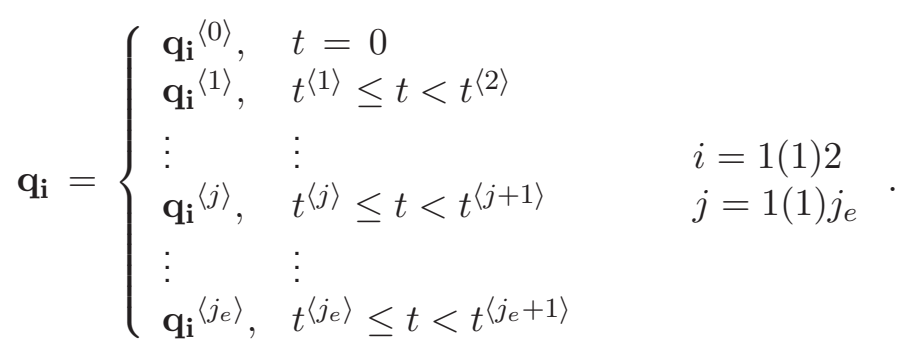

Thus, the state variables $\mathbf{q}_{1}, \dot{\mathbf{q}}_{1}$ and $\mathbf{q}_{2}, \dot{\mathbf{q}}_{2}$ are known at time $t$. The state variables $\mathbf{u}_{\mathbf{i}}, \dot{\mathbf{u}}_{\mathbf{i}}$ for the translatory DOFs are included herein. 
Dealing with non-smooth dynamic systems within FE methods, a fundamental challenge consists in finding an appropriate problem-oriented procedure of time integration. This crucial problem is associated with the accuracy of detecting the switching times (contact times) of the non-smooth system to be integrated [12]. In general, in view of the numerical time integration with a certain discrete time step $\Delta t$ only a limited degree of accuracy can be achieved in regard to determine the contact times. This leads to permanent numerical disturbances in the course of the motion. The undisturbed problem is orbitally stable as long as these disturbances remain below a critical limit. Thus, a sufficiently small time step $\Delta t$ is needed for an orbital stable solution. The present contribution will not focus to the problem of an appropriate time integration. Here, the standard $\mathrm{N}$ EWMARK scheme $\left(2 \alpha_{N}=\beta_{N}=1 / 2\right)$ with small constant time step $\Delta t$ is adopted as the number of DOFs is rather small though the central difference scheme may also be appropriate (cp. [11]). Mainly the problem of an appropriate contact formulation will be discussed in the following.

Suppose that the system contains $g_{n}$ possible pairs of contact nodes, then the actual state of contact is given by a set of indices $\Gamma_{c}$ from all $g_{c}$ contact points, which are closed at present time $t$. To simplify matters, the contact condition is only checked at the element nodes. Hence, an appropriate switching decision for the occurrence of a contact is given by the condition of a vanishing relative displacement on the corresponding contact nodes $\left(U_{k} \equiv 0\right)$. In the considered case of a time-discrete system with constant time steps $\Delta t$, a contact event is not captured with an arbitrary accuracy. Thus, the switching condition must be extended to

$$
U_{k}=u_{2, k}-u_{1, k} \leq 0, \quad \forall k \in \Gamma_{c}
$$

valid for each pair of contact nodes $k$. Then the state of contact on actual time $t$ reads

$$
\begin{array}{ll}
\Gamma_{n}=\left\{1(1) g_{n}\right\} & \text { possible contact points, } \\
\Gamma_{c}=\left\{k \in \Gamma_{n} \mid U_{k} \leq 0\right\} & \text { actually closed contact points (with } g_{c} \text { elements) }
\end{array}
$$

In the case that $\Gamma_{c} \neq \oslash$, at least one contact occurs at actual time $t$. Thus, the time $t$ denotes the separation time $t^{\langle j\rangle}$ for the beginning of the subsequent part of solution. To obtain the initial conditions for the velocities on the impacting nodes, the law of impact must be appraised. NEWTON's assumption of a sudden impact yields for the $k$ pairs of contact nodes

$$
\begin{aligned}
& \left.\dot{\mathbf{u}}_{\mathbf{1}}^{\left\langle t_{j}\right\rangle}\left(t_{j}+0\right)=\dot{\mathbf{u}}_{\mathbf{1}}^{\left\langle t_{j}\right\rangle}\left(t_{j}-0\right)+\left(\mathbf{I}+\mathbf{e}^{\left\langle t_{j}\right\rangle}\right) \mathbf{M}_{\mathbf{2}}^{*}\left\langle t_{j}\right\rangle\left(\mathbf{M}_{\mathbf{1}}^{*}\left\langle t_{j}\right\rangle+\mathbf{M}_{\mathbf{2}}^{*}\left\langle t_{j}\right\rangle\right)\right)^{-1} \dot{\mathbf{U}}^{\left\langle t_{j}\right\rangle}\left(t_{j}-0\right), \\
& \left.\dot{\mathbf{u}}_{\mathbf{2}}^{\left\langle t_{j}\right\rangle}\left(t_{j}+0\right)=\dot{\mathbf{u}}_{\mathbf{2}}^{\left\langle t_{j}\right\rangle}\left(t_{j}-0\right)-\left(\mathbf{I}+\mathbf{e}^{\left\langle t_{j}\right\rangle}\right) \mathbf{M}_{\mathbf{1}}^{*}\left\langle t_{j}\right\rangle\left(\mathbf{M}_{\mathbf{1}}^{*}\left\langle t_{j}\right\rangle+\mathbf{M}_{\mathbf{2}}^{*}\left\langle t_{j}\right\rangle\right)\right)^{-1} \dot{\mathbf{U}}^{\left\langle t_{j}\right\rangle}\left(t_{j}-0\right)
\end{aligned}
$$

with

$$
\begin{aligned}
& t_{j} \quad \text { present point in time (time step } j \text { ), } \\
& \dot{\mathbf{u}}_{\mathbf{i}}^{\left\langle t_{j}\right\rangle}\left(t_{j}+0\right)=\left[\dot{u}_{i, k}\left(t_{j}+0\right)\right] \quad \text { nodal velocities of the contacting masses } \\
& \text { after impact at the present time } t_{j} \text {, } \\
& \dot{\mathbf{u}}_{\mathbf{i}}^{\left\langle t_{j}\right\rangle}\left(t_{j}-0\right)=\left[\dot{u}_{i, k}\left(t_{j}-0\right)\right] \quad \text { nodal velocities of the contacting masses } \\
& \dot{\mathbf{U}}^{\left\langle t_{j}\right\rangle}\left(t_{j}-0\right)=\left[\dot{U}_{i, k}\left(t_{j}-0\right)\right] \quad \begin{array}{l}
\text { before impact at the present time } t_{j}, \\
\text { relative velocities of the contacting masses }
\end{array} \\
& \text { before impact at the present time } t_{j} \text {, } \\
& \text { I identity matrix, } \\
& \mathbf{e}^{\left\langle t_{j}\right\rangle}=\operatorname{diag}\left(\left[e_{k}\right]\right) \quad \text { matrix of the restitution coefficients for the } \\
& \mathbf{M}_{\mathbf{i}}^{*}\left\langle t_{j}\right\rangle=\operatorname{diag}\left(\left[m_{i, k k}^{*}\right]\right) \quad \text { matrix of the impacting masses } \\
& \begin{array}{l}
\text { matrix of the restitution coefficients for the } \\
\text { impacting masses at the present time } t_{j},
\end{array} \\
& \text { at the present time } t_{j} \text {, } \\
& \begin{array}{l}
i=1(1) 2 \\
\forall k \in \Gamma_{c}\left(t_{j}\right)
\end{array} \\
& \begin{array}{l}
i=1(1) 2 \\
\forall k \in \Gamma_{c}\left(t_{j}\right)
\end{array}
\end{aligned}
$$


Herein $m_{1, k k}^{*}, m_{2, k k}^{*}$ denote the masses affecting the impacting nodes at time $t_{j}$, which can be estimated from the corresponding translatory DOFs in the diagonalized mass matrix $\mathbf{M}_{\mathbf{i}}^{*}\left\langle t_{j}\right\rangle$. Furthermore, the diagonal matrix e contains the $g_{c}$ coefficients of restitution $e_{k}$. In the following, the restitution coefficient is assumed to be constant for all pairs of contact nodes. Then, only one parameter $e$ is needed, which is known from the preceding experimental investigation.

The initial conditions relating to the displacements $u_{1, k}^{\left\langle t_{j}\right\rangle}\left(t^{\langle j\rangle}+0\right), u_{1, k}^{\left\langle t_{j}\right\rangle}\left(t^{\langle j\rangle}+0\right)$ are given by the fact that the position of the contact nodes remains constant during the impact

$$
\begin{aligned}
& u_{1, k}^{\left\langle t_{j}\right\rangle}\left(t_{j}+0\right)=u_{1, k}^{\left\langle t_{j}\right\rangle}\left(t_{j}-0\right), \\
& \left\langle t_{j}\right\rangle \\
& u_{2, k}\left(t_{j}+0\right)=u_{2, k}^{\left\langle t_{j}\right\rangle}\left(t_{j}-0\right),
\end{aligned} \quad \forall k \in \Gamma_{c}\left(t_{j}\right) .
$$

For the very beginning of the motion at $t=0$, the displacements $\mathbf{q}_{1}^{\langle 0\rangle}, \mathbf{q}_{2}^{\langle 0\rangle}$ describe the stress-free, curved state of both laminae. The velocities $\dot{\mathbf{q}}_{1}^{\langle 0\rangle}, \dot{\mathbf{q}}_{2}^{\langle 0\rangle}$ are set equal to zero.

To get a stationary state of oscillation, a fairly long integration time of some hundred periods of excitation are necessary. The crucial problem is to detect a contact event $U \equiv 0$ as accurately as possible. Therefore, the applied switching condition $U \leq 0$ (eq. 12) must exhibit at most a weak inequality. Because constant time steps $\Delta t$ are used, the correct time of a contact lies in the range between $t_{j}$ and $t_{j+1}$ (with $t_{j+1}=t_{j}+\Delta t$ ). The above assumptions that the occurrence of contact events are always observed at the end of a time interval causes permanent numerical disturbances, which can totally distort the solution [12]. Therefore, the time step $\Delta t$ must be chosen sufficiently small compared to the period of excitation $T$.

The described scheme does not allow phases of motion showing permanent contact of a certain contact point. Instead, a sequence of contacts will occur.

In the following, all computations are performed with a discretization of 20 beam elements for each lamina. A constant time step $\Delta t=\frac{T}{1000}$ is taken. According to the experimental results, the coefficient of restitution is chosen as $e=0.10$. As mentioned before, it is assumed to be constant for all possible contact points. The length of the laminae, the cross-sections and the initial stressfree state are adopted from the real system. This gives the flexural rigidities $(E I)_{1}=1.51 \mathrm{Nm}^{2}$ for the thick lamina and $(E I)_{2}=0.16 \mathrm{Nm}^{2}$ for the thin lamina. Moreover, the material damping $D=0.008$ is taken from the experiment, which was determined by the decrease of the amplitudes of the free vibration separately arranged on each lamina. Both laminae show nearly identical amounts of damping. The small damping $D$ is included as RAYLEIGH damping. The amplitude of excitation $A=2.5 \mathrm{~mm}$ is fixed for all cases considered.

As a computation test for the proposed procedure, the experimental result given in Figure 3 [c] with a frequency of excitation $f=3.25 \mathrm{~Hz}$ is chosen as a reference. To confront the numerical results with the experimental ones, phase portraits of both nodes at the tip of each beam are considered (see Fig. 9). The phase curve depicts a time interval of 10 periods of excitation. Taking velocities into account in addition to the displacements gives proof of the stationary state of the solution.

Comparing the numerical results (Fig. 9 [b]) with the experimental ones (Fig. 9 [a]) shows sufficient conformity in the global shape as well as in the amplitudes of the displacement and the velocities. The chosen time step $\Delta t$ gives orbitally stable results. In numerical simulation the distinct phase of permanent contact is captured by numerous single impacts. This phenomenon observed in Figure 9 [b] needs to be explored in more detail (see Fig. 10 [a]). In those time intervals only a qualitative agreement of the numerical solution can be achieved in view of the phase portrait, while the corresponding time-displacement diagram (Fig. $10[\mathrm{~b}]$ ) is hardly affected by successive impacts. 
[a]

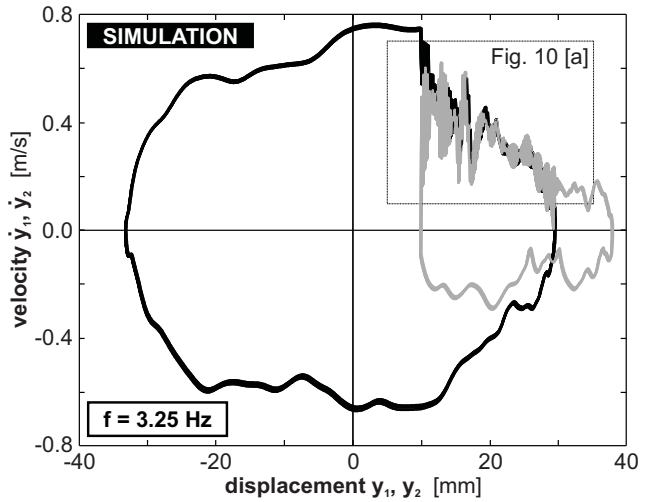

[b]

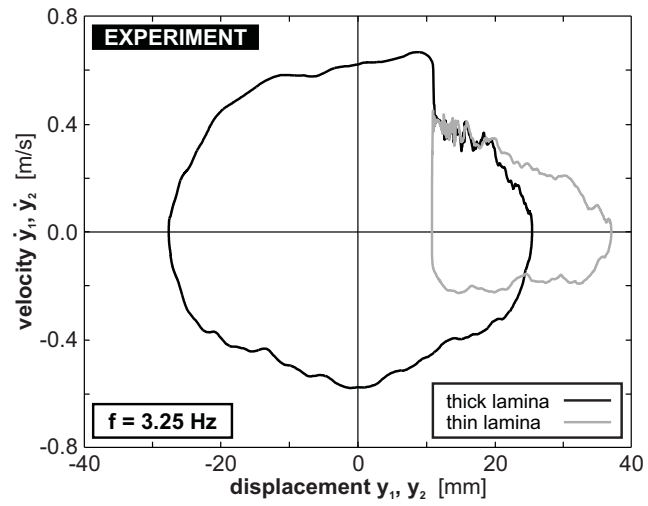

Fig. 9: Phase portraits for the stationary motion of both lamina $(f=3.25 H z)$ : [a] numerical result, [b] experimental result.

[a]
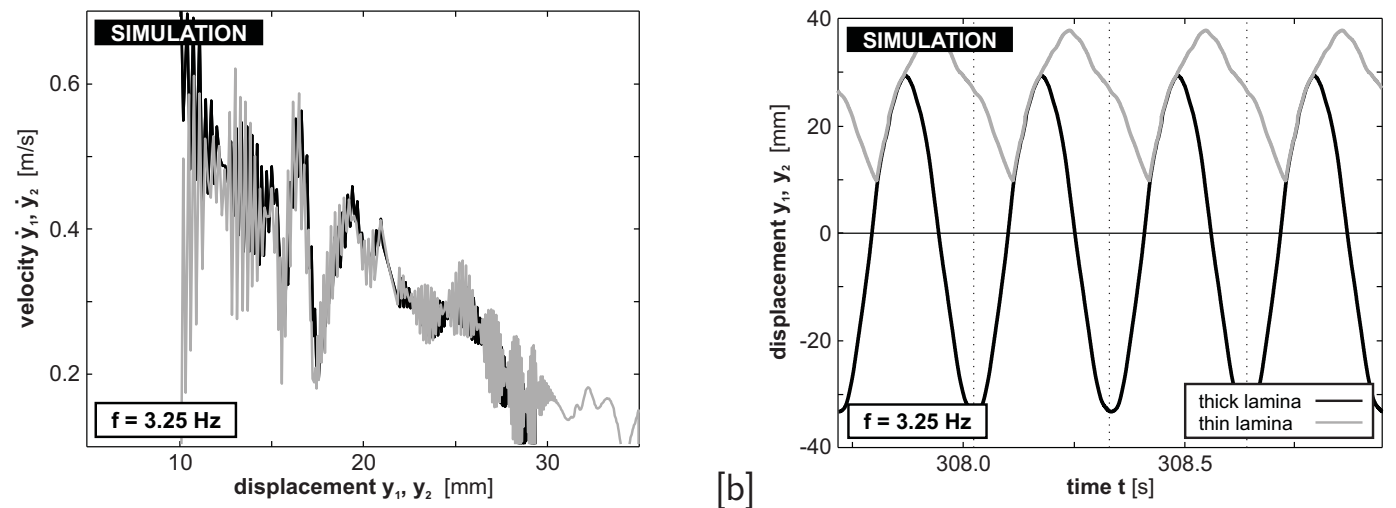

Fig. 10: Numerical simulation of the stationary motion of both lamina $(f=3.25 \mathrm{~Hz})$ : [a] detail of the phase portrait, [b] time history.

Summarizing the facts, NEWTON's impact law, which has been adopted for description of the dynamic contact-impact problem, captures the oscillation of the vibro-impacting system with sufficient accuracy. For this purpose, only one parameter must be adjusted to control the amount of energy loss. As a crucial advantage compared to the normally employed penalty method, the coefficient of restitution $e$ can be determined by experiments. The performed computations reveal an increased efficiency since no operations relating to the system matrices are necessary during the computation process. However, the drawback of this approach is that a permanent contact is separated into a sequence of single impacts. This fact may cause a reduction of the robustness of the solution in regard to the choice of parameters. To handle this situation, in the following an extended approach is proposed that comprises the advantages of both procedures, NEWTON's impact law and penalty method.

\subsection{NeWTON's impact law combined with penalty stiffness for description of dissi- pative contact}

Regularization of strong contact conditions by penalty method is one of the most common procedures for contact description within FE methods (e.g. [8]). This method involves introducing a contact stiffness $k_{c o n}$ and, in the case of dynamic contact, contact damping. In contrast to applying a law 
of impact with vanishing contact duration, the penalty method supposes a phase of permanent contact with minimal duration of one time step $\Delta t$ for each contact event. Thus, a partial state of permanent contact is included herein. As a main advantage for computation, this regularization allows a description of permanent contact while the number of DOFs is kept unaltered in comparison to the freely vibrating system.

In the following, the two methods are connected in a way that permits both a sudden impact and a motion in permanent contact. Therefore, the law of impact is employed to capture the impactlike contact phenomena showing a sudden change of the velocities as well as to observe energy dissipation during contact. Contact stiffness is only detached from the penalty method. In the case of permanent contact, this stiffness is adopted. Appropriate switching decisions for three several partial states, namely separated motion, sudden impact and permanent contact, are subsequently considered. Initially, two parameters are required (coefficient of restitution $e$, contact stiffness $k_{\text {con }}$ ), whereas the range of the main parameter $e$ can be determined by experiments. Since contact dissipation is solely captured by the law of impact, the two parameters are almost independent from one another, providing an additional advantage compared to the classical penalty method.

As previously mentioned, the system is governed by three partial states in motion. Again, the actual state of contact at present time $t$ can be observed by several sets of indicies. For this purpose, eq. 13 must be extended to capture all possible cases:

$$
\begin{aligned}
& \Gamma_{n}=\left\{1(1) g_{n}\right\} \\
& \Gamma_{c}=\left\{k_{c} \in \Gamma_{n} \mid U_{k_{c}} \leq 0\right\} \\
& \Gamma_{s}=\left\{k_{s} \in \Gamma_{c} \mid \dot{U}_{k_{s}} \geq \gamma \wedge\left(k_{s} \notin \Gamma_{p 0}\left(t_{j-1}\right) \vee k_{s} \notin \Gamma_{p p}\left(t_{j-1}\right)\right)\right\} \\
& \Gamma_{p 0}=\left\{k_{p 0} \in \Gamma_{c} \mid \dot{U}_{k_{p 0}}<\gamma \wedge k_{p 0} \notin \Gamma_{p p}\left(t_{j-1}\right)\right\} \\
& \Gamma_{p p}=\left\{k_{p p} \in \Gamma_{c} \mid k_{p p} \in \Gamma_{p 0}\left(t_{j-1}\right) \vee k_{p p} \in \Gamma_{p p}\left(t_{j-1}\right)\right\}
\end{aligned}
$$

possible contact points,

actually closed contact points, (with $g_{c}$ elements)

sudden impact with immediate separation (with $g_{s}$ elements),

beginning of permanent contact (with $g_{p 0}$ elements),

persistent permanent contact (with $g_{p p}$ elements) .

The condition for the occurrence of a contact event $U_{k_{c}} \leq 0$ can be kept unmodified in relation to eq. (12). Therefore, the law of impact given by eq. (14) must be applied on all pairs of contact nodes contained in the sets of indicies $\Gamma_{s}, \Gamma_{p 0}$. Thus, a sudden impact marks the beginning of each contact event. In the case of a vanishing translatory, relative velocity $\dot{U}_{k_{s}}=0$, checked at each pair of contact nodes contained in $\Gamma_{c}$, a partial state of permanent contact follows for these contact nodes. For all non-vanishing coefficients of restitution $e$, this switching condition can lead to a sequence of numerous impacts [9] as known from the previous section. To avoid these phenomena, a small threshold $\gamma$ for the relative velocity is introduced to assign the beginning of motion in permanent contact. Therefore, the weak inequality

$$
\dot{U}_{k}=\dot{u}_{2, k}-\dot{u}_{1, k}<\gamma, \quad \forall k \in \Gamma_{p 0}, \quad \gamma \ll 1
$$

allows the decision on whether a state of permanent contact follows on impact. In this case, the penalty stiffness $k_{c o n}$ is added on the corresponding contact nodes indicated by the set of index $\Gamma_{p 0}$.

$$
\mathbf{K}_{\mathbf{k}_{\mathbf{p} \mathbf{0}}}\left(t^{\langle j\rangle}+0\right)=\mathbf{K}_{\mathbf{k}_{\mathbf{p} \mathbf{0}}}\left(t^{\langle j\rangle}-0\right)+\left[\begin{array}{cc}
+k_{\text {con }} & -k_{\text {con }} \\
-k_{\text {con }} & +k_{\text {con }}
\end{array}\right], \quad \forall k_{p 0} \in \Gamma_{p 0}
$$


Herein, $\mathbf{K}_{\mathbf{k}_{\mathbf{p} 0}}$ denotes the matrix of stiffness for the corresponding pair of contact nodes $k_{p 0}$, that is involved as a submatrix in the total stiffness matrix $\mathbf{K}$

$$
\mathbf{K}=\left[\begin{array}{cc}
\mathbf{K}_{1} & \mathbf{0} \\
\mathbf{0} & \mathbf{K}_{2}
\end{array}\right]
$$

by summarizing both subsystems. It is evident from the structure of matrix $\mathbf{K}$, that the subsystems of equations (eq. (5)) are solely coupled by introducing the penalty term if permanent contact occurs. During a phase of permanent contact a vanishing relative velocity between the contact nodes is assumed. Contact stiffness $k_{\text {con }}$ for the corresponding pair of contact nodes remains in the system as long as the separation condition

$$
U_{k}=u_{2, k}-u_{1, k}>0 \quad k \in \Gamma_{p p} .
$$

is not satisfied. The condition is checked on each pair $k_{p p}$ contained in the set of indicies $\Gamma_{p p}$. In $\Gamma_{p p}$, all pairs of contact nodes are collected with the attribute of permanent contact at previous time $t_{j-1}$. Despite the fact that those pairs fulfill the contact condition (12), the law of impact must not be applied since there is persistent permanent contact.

Figure 11 briefly depicts the scheme of integration that involves the previously described contact algorithm combining NEWTON's impact law and penalty stiffness.

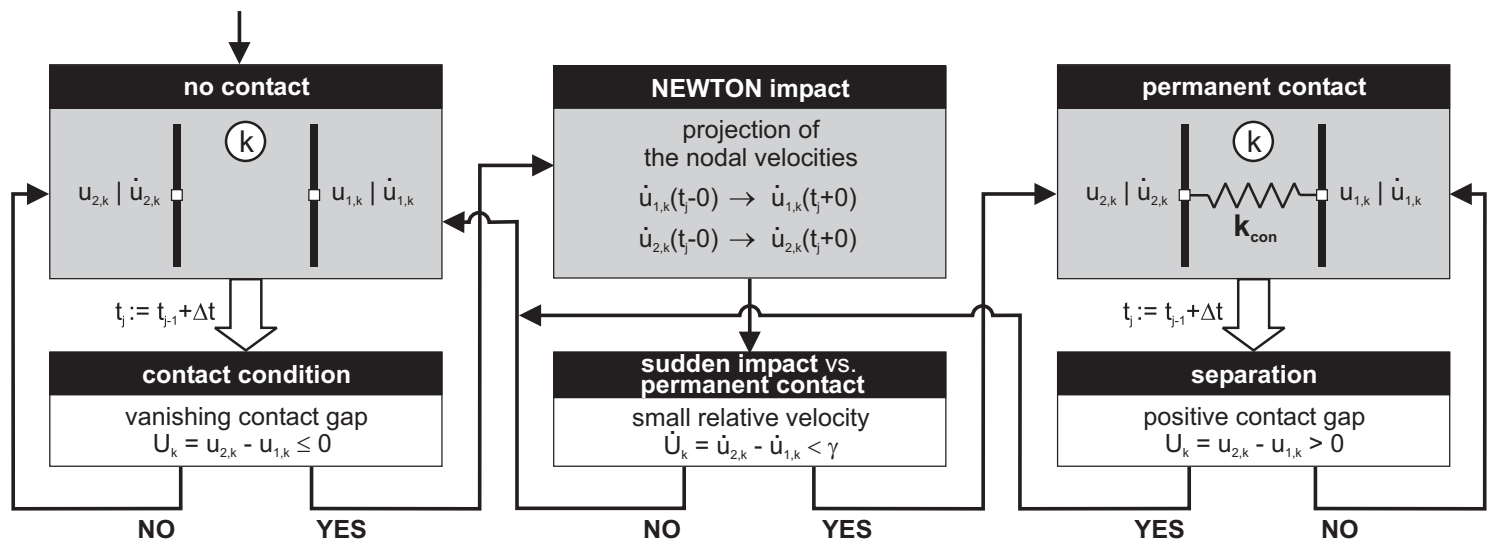

Fig. 11: Flow chart of the contact algorithm for the $k$-th pair of contact nodes.

In the following, several computations are presented for different types of oscillation whose corresponding experimental results are given in Figures $3[\mathrm{~b}]-[\mathrm{d}]$.

All parameters used for the previous computations (see section 3.1) are kept unaltered. In particular, the main contact quantity $e=0.10$ is preserved. Here, two additional numerical parameters have to be chosen: the penalty stiffness $k_{\text {con }}$ and the threshold $\gamma$ for permanent contact. Contact stiffness is set as $k_{\text {con }}=100$, which is reasonable in this analysis. The threshold of the relative velocity is chosen in such a way that $\gamma=0.5 \cdot 10^{-} 3$ is close to the thousandth part of the maximum relative velocity. These values are fixed for all cases considered in the following. The size of the maximum relative velocity can be captured approximately, for example, by considering the previous computations that are only based on the impact law.

To provide a complete view, additional computations must be mentioned here to reveal the robust character of the threshold value $\gamma$ for the treated system without generality for several problems. Obviously, larger values of $\gamma$ result in the fact that most of contacts are followed by an assumed state of permanent contact with duration of at least one time step $\Delta t$. Such a choice of $\gamma$ mostly 

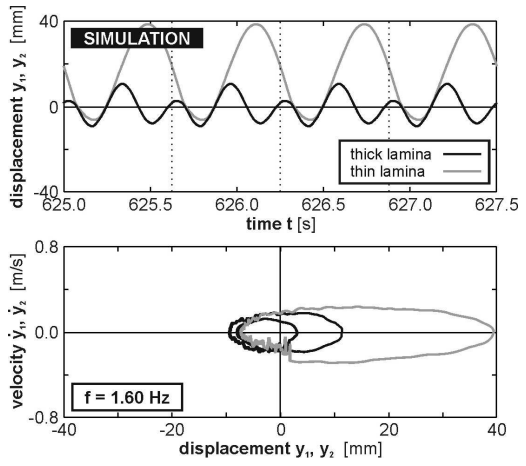

[a1]

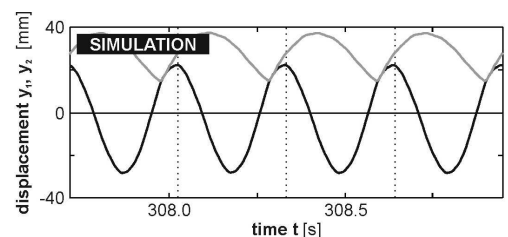

[b1]

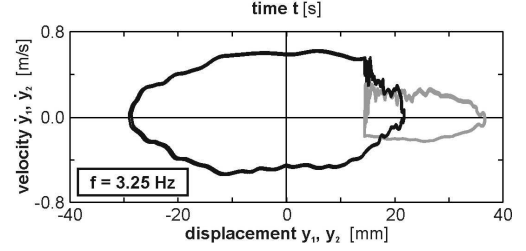

[b2]

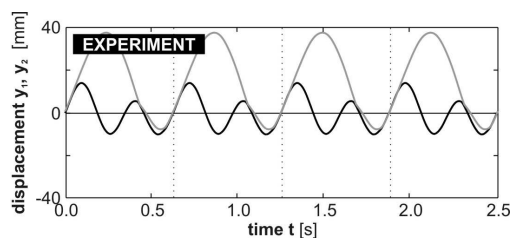

[a2]
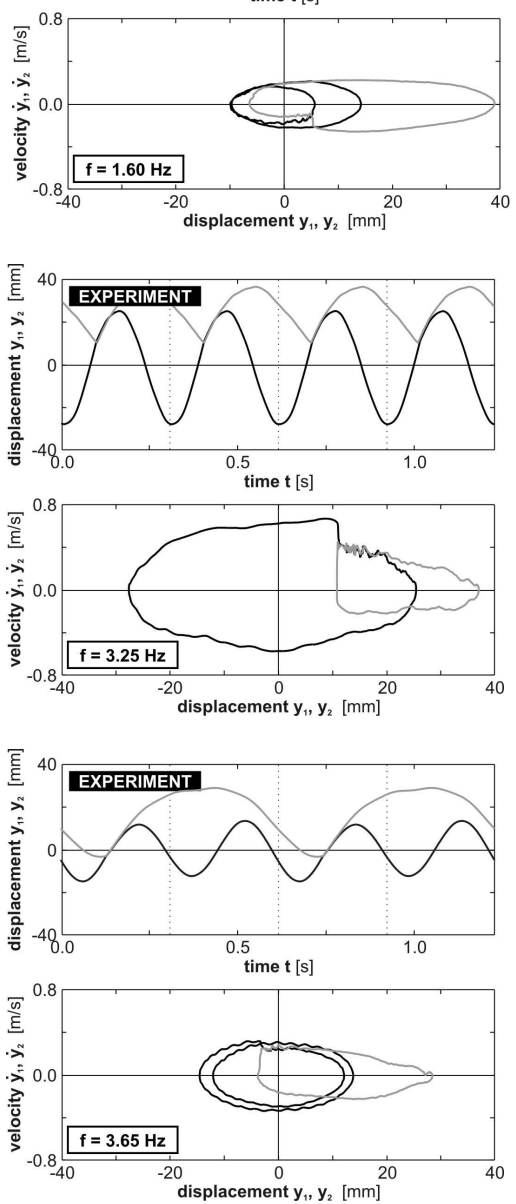

[c1]
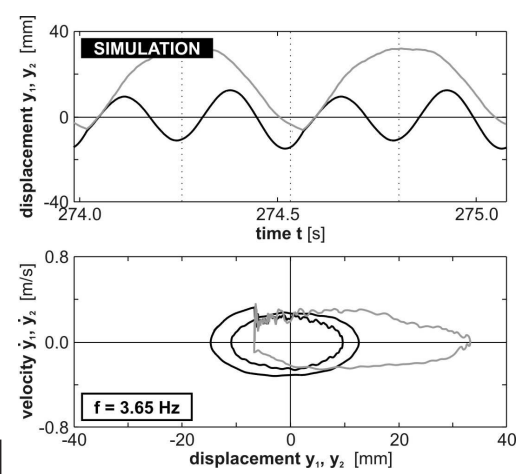

[c2

Fig. 12: Time history and phase portrait for the stationary motion of both lamina for various excitation frequencies, [a1]-[c1] numerical results $(e=0.10)$, [a2]-[c2] experimental results.

induces slight differences in relation to sudden impacts.

Again, several phase diagrams containing numerical results that correspond to the experimentally gained time-histories reported in Figure 3 [b]-[d] are employed to appraise the performance of the outlined contact description. Each phase curve is made up of a time interval of 10 periods of excitation. In addition, time histories of displacements with a length of 4 excitation periods are shown. Considering the system responses, in all cases good agreement of experimental and numerical results can be noted in relation to both the amplitudes and the shape of oscillation. In particular, the time histories of displacements are captured more precisely, which is of importance since future investigations on delaminated structures will primarily utilize this kind of response. Furthermore, the phase portraits show that in all cases an orbital stable solution is obtained. A distinct improvement is achieved in respect to the smoothness of simulation results during free vibration phase. Figure 13 illustrates the time interval during the contact event in more detail. By involving the partial state of permanent contact, more realistic contact behavior is achieved (see Fig. 12 [b2]) compared to the 
results of Figure $10[\mathrm{a}]$.

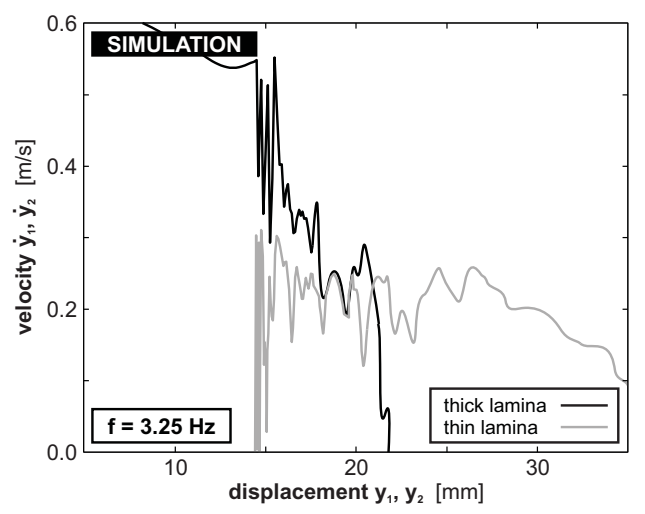

Fig. 13: Detail of phase portrait for the stationary motion of both lamina, $f=3.25 \mathrm{~Hz}$.

However, the discretization of the evoluting contact line by contact nodes may have caused the remaining variation of the oscillation in comparison to the experiments. This fact is supported by additional computations based on a finer discretization. Figure 14 exemplarily gives the result for 50 beam elements per lamina (case: $f=3.25 \mathrm{~Hz}$ ) leading to closely spaced contact nodes. All other parameters are identical. As evident from Figure 14, the content of higher frequencies in the velocity response is considerably reduced. Nevertheless, a substantially increase in the calculation time has to be accepted.

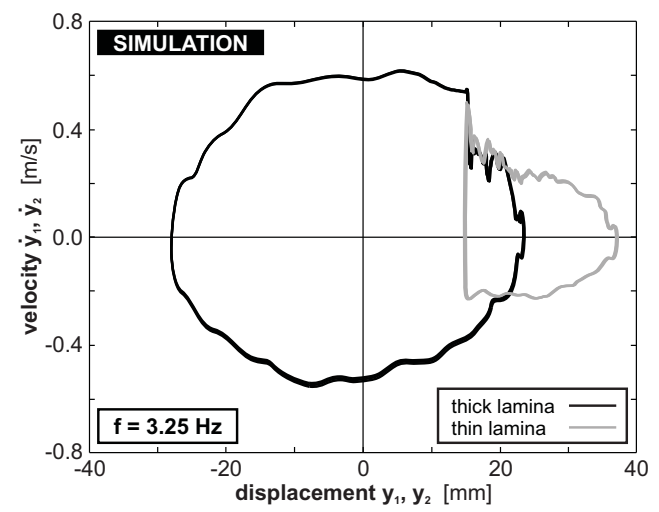

Fig. 14: Phase portraits for the stationary motion of both lamina based on 50 elements per beam, $f=3.25 \mathrm{~Hz}$.

In addition, the assumption that every contact is initiated by a sudden change of the velocity, which is not unreservedly valid for each contact event, includes some influence on the simulation result.

Despite these facts, the main advantages over the classical penalty method are evident from this investigation. First, the main parameter $e$ is experimentally determinable. The additionally required penalty stiffness $k_{c o n}$ exhibits in this connection a very limited influence on the result since the initial impact mainly controls the overall development of the contact due to its dissipative component. Moreover, the chosen set of parameters is applicable without modification to describe all treated types of motion that exhibit a wide variety. Such behavior cannot be expected from the springdamper regularization by penalty method as has been shown in [10]. 


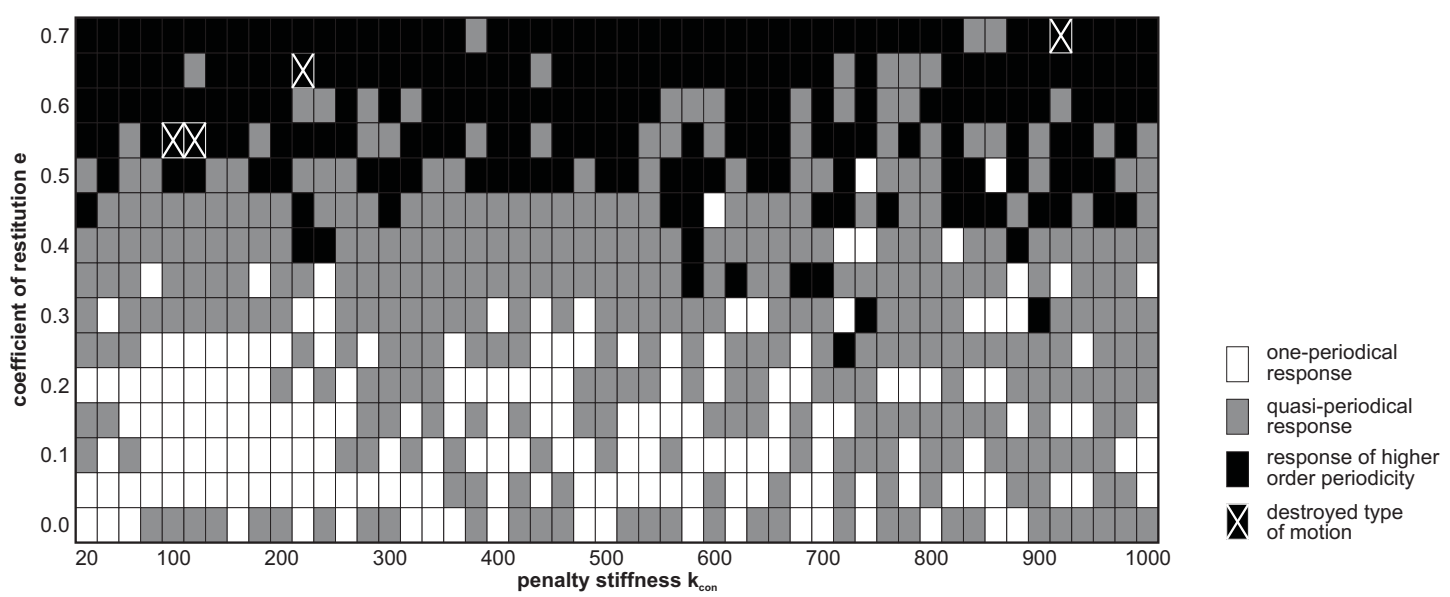

Fig. 15: Parametric plane for sets of parameters $e$ and $k_{c o n}, f=3.25 \mathrm{~Hz}$.

Furthermore, the described contact formulation shows a remarkable robustness relating to the choice of parameters $e, k_{c o n}$. For example Figure 15 contains a parametric plane that separates regions containing the correct type of solution from regions of distorted types of motion arising from an incorrect set of parameters. For this purpose, the numerical results computed for certain sets of parameters $e, k_{\text {con }}$ with a fixed excitation frequency $f=3.25 \mathrm{~Hz}$ have been categorized into oneperiodical response (correct type of solution), quasi-periodical responses (slightly distorted solution) and oscillations of higher order periodicity (distinctly disturbed solution). However, apart from a few exceptions, the basic type of response denoted by one contact event in one excitation period is preserved for all cases considered. When estimating the influence of the two parameters, the restitution coefficient mainly affects the solution in the treated range. Compared to the high sensitivity observed on classical penalty-regularization [11], the presented approach seems to provide a promising alternative due to its robustness.

\section{Conclusions}

Forced oscillations of delaminated structures are dominated by continuously evoluting impact-like contacts. First, the arising contact phenomena are studied experimentally on a realistic model situation for the delamination problem. This continuous vibro-impacting system shows a broad variety of possible types of oscillation. Depending on the frequency of excitation, a bifurcation scenario occurs. Those phenomena, previously known from punctual impacting systems, are even significant properties of continuously contacting oscillators. The distortion factor quantifies the nonlinearity of the system's response at certain excitation frequencies.

Several basic experiments were carried out to characterize the properties of lateral impacts on beamtype structures that provide much information for understanding this special type of contact. As a main result, a vanishing contact time compared to the time scale of the oscillation was found. Moreover, these experiments reveal a distinct amount of energy dissipation during the contact.

Armed with this knowledge, an appropriate contact description for numerical simulation using Finite Element methods is addressed mainly based on the theory of sudden impacts involving contact dissipation. In contrast to the classical penalty method, the main parameter, namely the coefficient of restitution, can be determined easily by experimental means. Computational results document the robustness of the procedure in relation to the choice of parameters. In most all if the cases conside- 
red above, the type of the experimental reference solution was preserved numerically within a widely open range of parameters. Furthermore, the set of parameters can be kept constant for all of the very different types of motion previously considered. Based on this robustness, the present technique turns out to be very advantageous and will be beneficial to further development of reliable methods for model-based damage detection employing clapping phenomena.

\section{References}

[1] Farrar, C. R.; Sohn, H.; Fugate, M. L.; Czarnecki, J. J.: Integrated structural health monitoring. SPIE's 8th Annual International Symposium on Smart Structures and Materials, Newport Beach, (2001).

[2] Zou, L.; Tong, L.; Steven G. P.: Vibration-based model-dependent damage (delamination) identification and health monitoring for composite structures. Journal of Sound and Vibration, 230(2): 357-378, (2000).

[3] Zheng, Y.; Maev, R. G.; Solodov, I. Y.: Nonlinear acoustic applications for material characterization: A review.

Canadian Journal of Physics, 77: 927-967 (1999).

[4] Vielsack, P.: A vibro-impacting model for the detection of delamination. Journal of Sound and Vibration, 253(2): 347-358, (2002).

[5] Philippow, E.: Grundlagen der Elektrotechnik. Akademische Verlagsgesellschaft Geest \& Portig KG / Leipzig, (1967).

[6] Hu, B.; Schiehlen, W.; Eberhard, P.: Comparison of analytical and experimental results for longitudinal impacts on elastic rods. Journal of Vibration and Control, 9: 157-174 (2003).

[7] Engleder, T.; Vielsack, P.; Schweizerhof, K.: F.E.-regularisation of non-smooth vibrations due to friction and impacts. Computational Mechanics, 28: 162-168 (2002).

[8] Zienkiewicz, O. C.; Taylor, R. L.: The Finite Element Method. Butterworth-Heinemann, Oxford, (2000).

[9] Valente, A. X.; McClamroch, N. H.; Mezi, I.: Hybrid dynamics of two coupled oscillators that can impact a fixed stop. International Journal of Non-Linear Mechanics, 38(5): 677-689 (2003).

[10] Müller, I.; Vielsack, P.: Penalty-regularisation of a dissipative vibro-impacting system. Journal of Computational and Applied Mechanics, 4(2): 173-186 (2003).

[11] Müller, I.; Konyukhov, A.; Vielsack, P.; Schweizerhof, K.: Parameter estimation for Finite Element analyses of stationary oscillations of a vibro-impacting System. Engineering Structures, 27(2): 191-201 (2005).

[12] Vielsack, P.; Hartung, A.: An example for the orbital stability of permanently disturbed non-smooth motions.

ZAMM 79(6): 389-397, (1999). 